\title{
Editorial: The Interplay Between Epigenetic Regulation and Other Cellular Processes
}

\author{
Kai Tang ${ }^{1 *}$ and Huiming Zhang ${ }^{2 *}$ \\ ${ }^{1}$ Department of Horticulture and Landscape Architecture, Purdue University, West Lafayette, IN, United States, ${ }^{2}$ Shanghai \\ Center for Plant Stress Biology, CAS Center for Excellence in Molecular Plant Sciences, Chinese Academy of Sciences, \\ Shanghai, China
}

Keywords: epigenetic regulation, cellular processes, DNA methylation, histone modification, RNA metabolism, lipid, folate, germ cells

\section{Editorial on the Research Topic}

\section{The Interplay Between Epigenetic Regulation and Other Cellular Processes}

Epigenetic changes can influence chromatin structure and, in turn, the accessibility of genetic information as well as the stability of the whole genome. As a result, epigenetic modifications are important to many biological processes, and disruption of epigenetic configuration can lead to developmental abnormalities in plants and mammals, such as failure in tomato fruit ripening

OPEN ACCESS

Edited by:

Rui Henrique,

Portuguese Oncology

Institute, Portugal

Reviewed by:

Alexander E. Berezin,

Zaporizhia State Medical

University, Ukraine

*Correspondence:

Kai Tang

tang58@purdue.edu

Huiming Zhang

hmzhang@psc.ac.cn

Specialty section

This article was submitted to Epigenomics and Epigenetics,

a section of the journal

Frontiers in Genetics

Received: 05 April 2021

Accepted: 13 April 2021

Published: 07 May 2021

Citation:

Tang $K$ and Zhang H (2021) Editorial:

The Interplay Between Epigenetic

Regulation and Other Cellular

Processes. Front. Genet. 12:691202.

doi: 10.3389/fgene.2021.691202 (Zhong et al., 2013; Lang et al., 2017) and embryo lethality in mice (Cortázar et al., 2011; Blewitt and Whitelaw, 2013). In addition to coordinating with developmental processes, epigenetic regulation can also play an important role in organisms' responses and adaptation to environmental changes (Etchegaray and Mostoslavsky, 2016; Zhang et al., 2018). Thus, epigenetic processes are tightly regulated in coordination with other cellular processes.

On one hand, cellular processes with important functions can be mediated by epigenetic modifications at the transcriptional level. For instance, Steadman et al. reported that algae cultures treated with 5-aza-2'-deoxycytidine, an inhibitor of DNA methylation, resulted in a remarkable increase in the level of lipid accumulation and increased cell size. Similarly, Zhang M. et al. discovered that DNA methylation regulates fatty acid metabolism and intramuscular fat deposition in chicken. As reviewed in Zhang $\mathrm{H}$. et al., histone deacetylation leads to the initiation and progression of osteoarthritis; while Li et al. showed that knockdown of SETDB1, a histone H3 lysine 9 (H3K9) methyltransferase, resulted in increased levels of reactive oxygen species and impaired proliferation of mouse spermatogonial stem cells.

On the other hand, epigenetic features can be affected by other important biological processes. Certain cellular processes are inherently required for epigenetic modifications, especially DNA methylation and histone post-transcriptional modifications, which are enzymatic processes that involve not only the chromatin but also donor molecules for the modifications. For instance, disruptions in the folate biosynthesis pathway impair the supply of methyl groups for DNA methylation and for histone methylation, resulting in transcriptional desilencing at certain genomic loci in Arabidopsis thaliana due to lowered levels of DNA methylation and histone H3K9 dimethylation (Zhang et al., 2012). In this Research Topic, González et al. revealed that, in mouse male germ cells, cocaine caused epigenetic reprogramming of histone modifications involved in gene silencing and the histone-to-protamine replacement, while the effects of cocaine on the histone modifications can be largely blocked by inhibition of the dopamine receptor 1 (DRD1), suggesting a novel connection between the DRD1-dependent dopaminergic system and epigenetic regulation. In Arabidopsis thaliana, Zhu et al. observed genome hypomethylation caused by chemical inhibition of the target of rapamycin (TOR), thereby pointing to a connection between 
epigenetic regulation and this evolutionarily conserved master regulator, which integrates multiple cellular processes to promote growth in all eukaryotes (Dobrenel et al., 2016). As reviewed by Ye et al., the transcription factor ZNF143, which shows higher expression in cancer cells than normal cells, connects promoters to distal regulatory elements and thereby mediates chromatin looping.

Many important biological processes involve RNA metabolism, such as $\mathrm{N}^{6}$-methyladenosine that carries many functions in plants as reviewed by Zheng et al., as well as the newly identified miRNAs that silence an important regulator of apoptosis in the report by Coccia et al. Epigenetic regulation of the chromatin are often closely related to RNA metabolism. As reviewed by Zhang J. et al., a crosstalk exists between epigenetic regulation and alternative RNA processing including alternative splicing and alternative polyadenylation. Apparently, the interplay between epigenetic regulation and certain cellular processes can be bidirectional.

The interplay between epigenetic regulation and diverse cellular processes has become increasingly valued over the past few years. While this theme is highlighted by the articles in this

\section{REFERENCES}

Blewitt, M., and Whitelaw, E. (2013). "The use of mouse models to study epigenetics." Cold Spring Harbor Perspectiv. Biol. 5:a017939. doi: 10.1101/cshperspect.a017939

Cortázar, D., Kunz, C., Selfridge, J., Lettieri, T., Saito, Y., MacDougall, E., et al. (2011). "Embryonic lethal phenotype reveals a function of TDG in maintaining epigenetic stability." Nature. 470, 419-423. doi: 10.1038/nature09672

Dobrenel, T., Camila, C., Johannes, H., Christophe, R., Michel, V., Bruce, V., et al. (2016). “TOR signaling and nutrient sensing." Annu. Rev. Plant. Biol. 67, 261-285. doi: 10.1146/annurev-arplant-043014-114648

Etchegaray, J. P., and Mostoslavsky, R. (2016). "Interplay between metabolism and epigenetics: a nuclear adaptation to environmental changes." Mol. Cell.62, 695-711. doi: 10.1016/j.molcel.2016.05.029

Lang, Z., Wang, Y., Tang, K., Tang, D., Datsenka, T., Cheng, J., et al. (2017). "Critical roles of DNA demethylation in the activation of ripening-induced genes and inhibition of ripening-repressed genes in tomato fruit." Proc. Natl. Acad. Sci. U. S. A. 2017:5233. doi: 10.1073/pnas.1705233114

Zhang, H., Xiangyang, D., Daisuke, M., Sean, C., Jee-Eu, O., and JianKang, Z. (2012). Sulfamethazine suppresses epigenetic silencing in
Research Topic, the need for a thorough understanding of the epigenetics-connected cellular network continues to urge more discoveries and new insights in this important research area.

\section{AUTHOR CONTRIBUTIONS}

All authors listed have made a substantial, direct and intellectual contribution to the work, and approved it for publication.

\section{FUNDING}

Research in HZ lab has been supported by the Chinese Academy of Sciences.

\section{ACKNOWLEDGMENTS}

We thank all authors who submitted their work for this Research Topic as well as the invaluable help of reviewers in manuscript evaluation and the support of professional Editorial staff at Frontiers.

Arabidopsis by impairing folate synthesis. Plant Cell. 24, 1230-1241. doi: 10.1105/tpc.112.096149

Zhang, H., Zhaobo, L., and Jian-Kang, Z. (2018). Dynamics and function of DNA methylation in Plants. Nat. Rev. Mol. Cell Biol. 19, 489-506. doi: 10.1038/s41580-018-0016-Z

Zhong, S., Fei, Z., Chen, Y. R., Zheng, Y., Huang, M., Vrebalov, J., et al. (2013). "Single-base resolution methylomes of tomato fruit development reveal epigenome modifications associated with ripening." Nat. Biotechnol. 31, 154-159. doi: 10.1038/nbt.2462

Conflict of Interest: The authors declare that the research was conducted in the absence of any commercial or financial relationships that could be construed as a potential conflict of interest.

Copyright (c) 2021 Tang and Zhang. This is an open-access article distributed under the terms of the Creative Commons Attribution License (CC BY). The use, distribution or reproduction in other forums is permitted, provided the original author(s) and the copyright owner(s) are credited and that the original publication in this journal is cited, in accordance with accepted academic practice. No use, distribution or reproduction is permitted which does not comply with these terms. 